\title{
Precise base editing with CC context- specificity using engineered human APOBEC3G-nCas9 fusions
}

\author{
Zhiquan Liü ${ }^{1 \dagger}$, Siyu Chen ${ }^{1 \dagger}$, Huanhuan Shan ${ }^{1}$, Yingqi Jia ${ }^{1}$, Mao Chen ${ }^{1}$, Yuning Song ${ }^{1}$, Liangxue Lai ${ }^{1,2,3,4^{*}}$ and \\ Zhanjun $\mathrm{Li}^{1 *}$ (D)
}

\begin{abstract}
Background: Cytidine base editors (CBEs), composed of a cytidine deaminase fused to Cas9 nickase (nCas9), enable efficient C-to-T conversion in various organisms. However, current base editors can induce unwanted bystander C-to-T conversions when multiple Cs are present in the $\sim 5$-nucleotide activity window of cytidine deaminase, which negatively affects their precision. Here, we develop a new base editor which significantly reduces unwanted bystander activities.

Results: We used an engineered human APOBEC3G (eA3G) C-terminal catalytic domain with preferential cytidine-deaminase activity in motifs with a hierarchy $C C C>C C C>C \subseteq$ (where the preferentially deaminated $\mathrm{C}$ is underlined), to develop an eA3GBE with distinctive CC context-specificity and reduced generation of bystander mutations. Targeted editing efficiencies of 18.3-58.0\% and 54.5-92.2\% with excellent Cㄷ context-specificity were generated in human cells and rabbit embryos, respectively. In addition, a base editor that can further recognize relaxed NG PAMs is achieved by combining hA3G with an engineered SpCas9-NG variant. The A3G-BEs were used to induce accurate single-base substitutions which led to nonsense mutation with an efficiency of $83-100 \%$ and few bystander mutations in Founder (F0) rabbits at Tyr loci.
\end{abstract}

Conclusions: These novel base editors with improved precision and Cㅡ context-specificity will expand the toolset for precise gene modification in organisms.

Keywords: CRISPR/Cas9, Base editor, eA3G, Precision

\section{Background}

The clustered regularly interspaced short palindromic repeat (CRISPR) system has exhibited powerful genome manipulation capability in various organisms [1]. Base editor, a revolutionary technology derived from the CRIS PR system, is composed of a cytidine deaminase or an evolved adenine deaminase fused to nCas 9 and enables the conversion of C.G to T.A or A.T to G.C base pair in organisms, respectively $[2,3]$. In contrast to conventional gene-editing nucleases, $\mathrm{CBE}$ represents significant

\footnotetext{
* Correspondence: lai_liangxue@gibh.ac.cn; lizj_1998@jlu.edu.cn

'Zhiquan Liu and Siyu Chen contributed equally to this work.

'Key Laboratory of Zoonosis Research, Ministry of Education, College of

Animal Science, Jilin University, Changchun 130062, China

Full list of author information is available at the end of the article
}

advances in precise genome manipulation since it can achieve targeted C-to- $\mathrm{T}$ conversions without generating DNA double-strand breaks (DSBs) or requiring a donor template, and it induces lower levels of unwanted insertion/deletion mutations (indels) $[2,4]$. The most commonly used $\mathrm{CBE}$ architecture, rA1-BE, consists of rat APOBEC1 (rA1) fused to a Streptococcus pyogenes Cas9 (SpCas9) nickase [2]. Efficient editing by rA1-BE requires the target $\mathrm{C}$ within a $\sim 5$-nucleotide window near the protospacer-adjacent motif (PAM)-distal end of the protospacer (positions $4-8$, counting the PAM as positions 21-23) in human cells [2]. The unwanted bystander C-to-T conversions will be generated when multiple Cs are present in the enzyme's activity window

C C The Author(s). 2020 Open Access This article is licensed under a Creative Commons Attribution 4.0 International License, which permits use, sharing, adaptation, distribution and reproduction in any medium or format, as long as you give appropriate credit to the original author(s) and the source, provide a link to the Creative Commons licence, and indicate if changes were made. The images or other third party material in this article are included in the article's Creative Commons licence, unless indicated otherwise in a credit line to the material. If material is not included in the article's Creative Commons licence and your intended use is not permitted by statutory regulation or exceeds the permitted use, you will need to obtain permission directly from the copyright holder. To view a copy of this licence, visit http://creativecommons.org/licenses/by/4.0/ The Creative Commons Public Domain Dedication waiver (http://creativecommons.org/publicdomain/zero/1.0/) applies to the data made available in this article, unless otherwise stated in a credit line to the data. 
$[2,5]$. It negatively affects the precision of targeted base editing, which are not ideal for precise disease modeling and gene therapy where accurate single $C$ substitution is required [6].

To overcome this limitation, optimized rA1 with mutant deaminase domains (YE base editors) or shortened linker between $\mathrm{rA} 1$ and $\mathrm{nCas} 9$ has been used to narrow the editing window in human cells $[7,8]$. The representative YE systems termed YE1 (W90Y+R126E) and YEE (W90Y+R126E+R132E), to effectively narrow the width of the editing window from $\sim 5$ nucleotides to as little as $\sim 1-2$ nucleotides in human cells [7]. Additionally, YEEBE showed better accuracy than YE1-BE, but has lowered editing efficiency at target loci $[7,9]$. Moreover, an engineered human APOBEC3A (eA3A) domain with TCR $(\mathrm{R}=\mathrm{A} / \mathrm{G})$ context-specificity has been reported to efficiently reduce bystander mutations, and it has been proven to be superior to conventional base editors with narrowed window in the TCR motifs $[9,10]$. Contextdependent base editors, such as eA3A-BE, represent an important direction that offers precise base editing, while the application of them was restricted by the presence of TCR motifs [9]. Additionally, although many precise base editors exist currently, it is still difficult to achieve accurate editing in target sites with multiple Cs.

Previous study has shown the hA3G preferentially deaminates cytidines in $\mathrm{C} \underline{\mathrm{C}}$ and $\mathrm{CC} \underline{\mathrm{C}}$ motif in vitro [1113]; thus, we speculated it would have the potential to be developed as a CC context-dependent base editor. In addition, hA3G-BE3 has been reported to induce C-to-T conversions in mammalian cells with lower efficiency compared with rA1-BE3 $[14,15]$, but no one has thoroughly evaluated its editing efficiency in CC context, for which it shows distinctive preference. Here, a new base editor, eA3G-BE, was developed for drastically reducing bystander mutations using an engineered hA3G Cterminal catalytic domain which preferentially deaminates cytidines in specific motifs according to a $\mathrm{CC} \underline{\mathrm{C}}>\mathrm{C} \underline{\mathrm{C}}>\mathrm{C} \underline{\mathrm{C}}$ hierarchy firstly (where the preferentially deaminated $\mathrm{C}$ is underlined). Moreover, the further engineered variant, eA3G-NG, could be used to expand genome-targeting scope with NG PAMs. These new base editing tools provide a simple and efficient method for inducing precise single-nucleotide substitution with $\mathrm{CC}$ context-specificity.

\section{Results}

Characterization of eA3G-BE that selectively edits Cs with CC context-specificity

The hA3G has a C-terminal catalytic domain (CTD) and a second pseudocatalytic domain at $\mathrm{N}$-terminal which retains the same tertiary fold, but is not catalytically active [11]. To make use of its beneficial properties for base editing, we replaced $\mathrm{rA} 1$ with the engineered $\mathrm{C}$ - terminal catalytic domain of hA3G (hA3G-CTD) in rA1BE4max [16], the current optimal architecture of $\mathrm{CBE}$, to create eA3G-BE4max (referred to as eA3G-BE) (Fig. 1a).

To evaluate its general efficacy, we firstly tested both rA1-BE and eA3G-BE at 7 target sites with multiple Cs in human cells by co-transfecting them with the respective single guide RNAs (sgRNAs) into HEK293T cells (Fig. 1b). Western blotting demonstrated the protein products of eA3G-BE was comparable to that of classical rA1-BE (Additional file 1: Fig. S1). Base editing frequencies were evaluated from Sanger sequence chromatograms using EditR, a robust and inexpensive base editing quantification software [17]. Our results suggested that, compared with rA1-BE system, eA3G-BE showed a distinct preference for $\mathrm{CC}$ context in all seven tested sites (Fig. 1c). In two-C context, the eA3G-BE system has similar or lower editing efficiency at the second C (CC) but significantly reduced $\mathrm{C}$-to- $\mathrm{T}$ conversions at the first $\mathrm{C}$ (CC) (Fig. 1c, d). Moreover, in more than three-C context, the eA3G-BE exhibited similar efficiency comparable to that of rA1-BE at the last $\mathrm{C}$ (CCC) (mean $37.0 \%$ vs. $28.8 \%, P=0.055)$, meanwhile with lower efficiency at the middle C (CCC) (mean $17.4 \%$ vs. $31.4 \%$, $P=0.0007)$ and significantly reduced efficiency at the first C (CCC) (mean $4.3 \%$ vs. $37.1 \%, P<0.0001$ ) (Fig. 1c, d). In addition, the significantly reduced base editing efficiencies of eA3G-BE were observed in 6 target sites with non-CC contexts compared with that of $\mathrm{rA1}-\mathrm{BE}$ (Additional file 1: Fig. S2). The eA3G-BE can efficiently induce base editing in a major window $(\sim 7 \mathrm{nt}$, positions 4-10 in the sgRNA target site) compared with rA1-BE ( $6 \mathrm{nt}$, positions $4-9$ ), and it can even edit distal C14 at Emx1-3 (Fig. 1e, f). Moreover, the eA3G-BE showed reduced off-target base editing compared with $\mathrm{rA1}-\mathrm{BE}$, consistent with previous report of eA3A-BE [9] (Additional file 1: Fig. S3). Taken together, these results suggested that the eA3G-BE can induce site-dependent lower or similar base editing efficiency compared to that of $\mathrm{rA1}-\mathrm{BE}$, meanwhile maintaining distinct preference for $\mathrm{CC}$ context in human cells.

\section{Comparison of base editing activities and precision among $\mathrm{eA} 3 \mathrm{G}-\mathrm{BE}$ and other precise BEs}

To compare the precision of eA3G-BE with current representative precise $\mathrm{BEs}$, we compared the editing activities of six base editor fusions at three representative target sites with multiple Cs: the original $\mathrm{rA1}-\mathrm{BE}$, the eA3G-BE, three rA1-BE variants, YE1 and YEE (YE BEs) which have mutations in $\mathrm{rA1}$ designed to slow its kinetic rate so as to restrict the editing window, $7 \mathrm{aa}-\mathrm{BE}$ with narrowed window by shortening the linker between the rA1 and the Cas9 domain, and eA3A-BE which bears N57G mutation in hA3A (Fig. 2a). Among the six base 


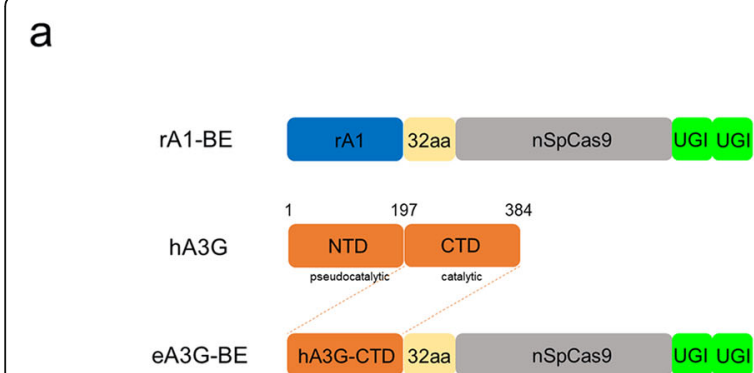

C
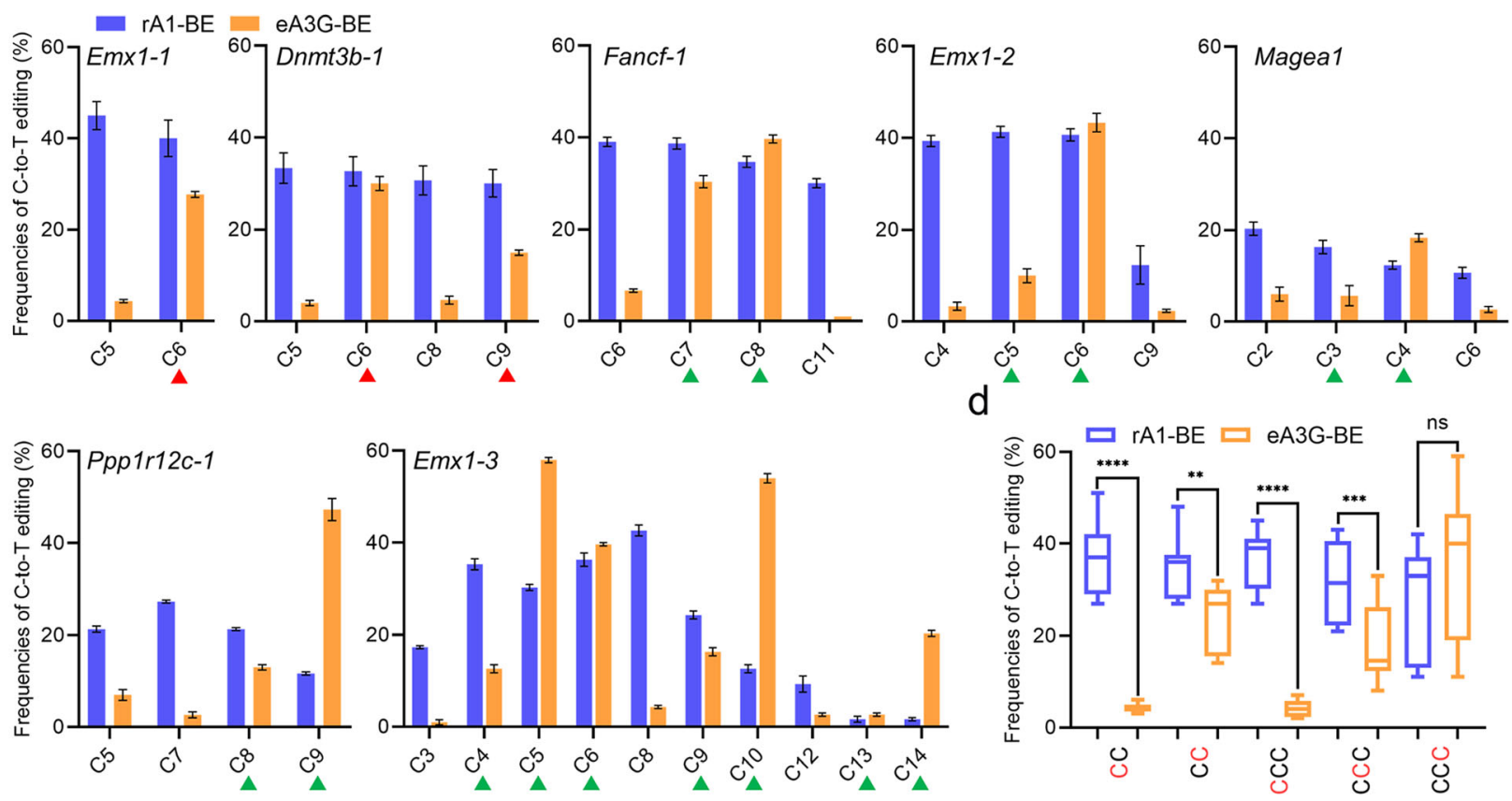

e

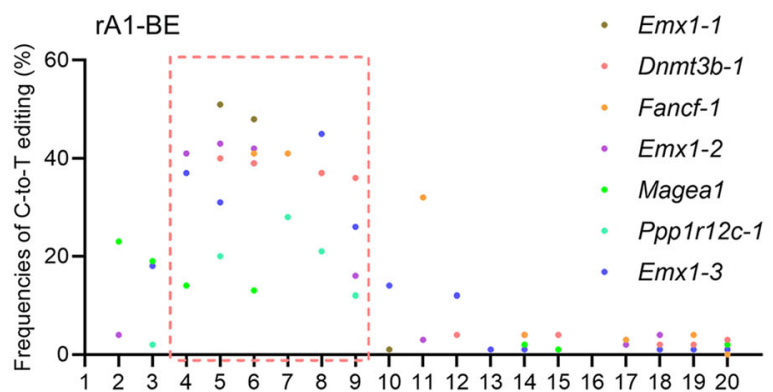

f

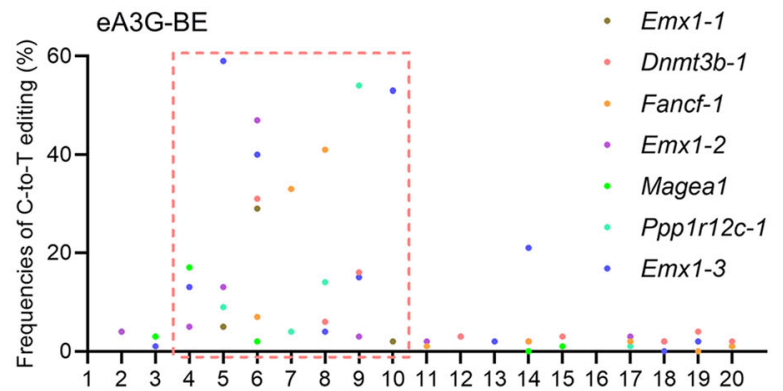

Fig. 1 Base editing with CC context-specificity mediated by eA3G-BE in human cells. a Schematic representation of rA1-BE and eA3G-BE architecture. $\mathbf{b}$ Protospacers and PAM (green) sequences of the genomic loci tested, with the target Cs shown in red. Cytosines are counted with the base distal to the PAM setting as position 1. c Summary of C-to-T editing frequencies induced by rA1-BE and eA3G-BE systems on each cytosine at seven target sites. The eA3G-BE showed obvious preference at the cytosines in a CC (red triangle) or CCC (green triangle) context. $\mathbf{d}$ Effect of sequence context on base

editing by $\mathrm{rA1}-\mathrm{BE}$ and $\mathrm{A}$ A3G-BE (window 4-9). The frequencies were calculated using the data in Fig. 2c. e, f Summary of the base editing frequency at each cytosine in the spacer region for the indicated 7 sgRNAs using rA1-BE (e) and eA3G-BE (f). These data show that the major editing window ranges of rA1-BE and eA3G-BE from positions 4 to 9 or from positions 4 to 10 in spacer region, respectively. Values and error bars reflect the mean \pm s.e.m. of three independent biological replicates 


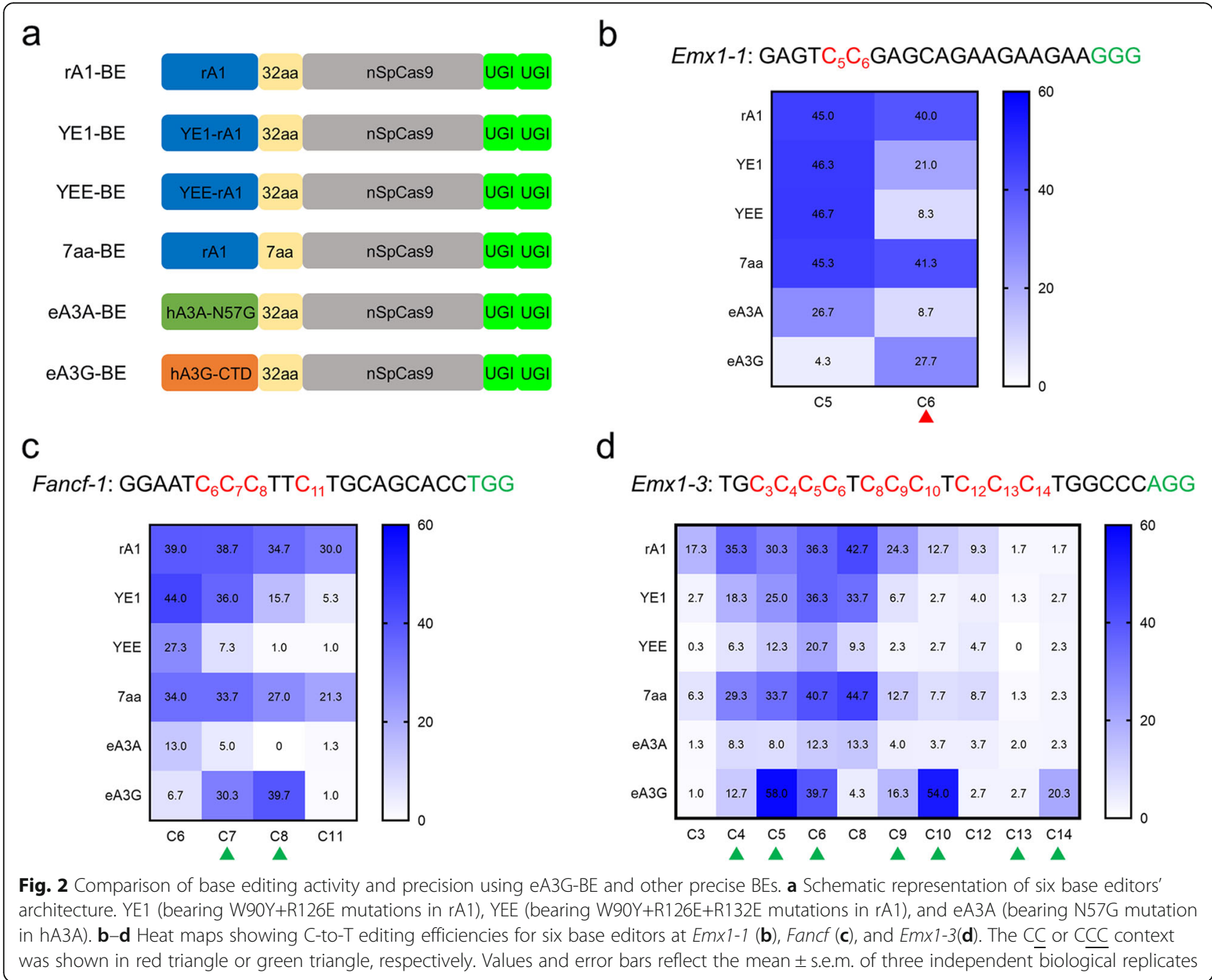

editors tested, eA3G-BE displayed the unique CC context-specificity, meanwhile minimizing bystander cytidine editing in non-CC context at all tested sites (Figs. 2b-d and Additional file 1: Fig. S4). Moreover, eA3G-BE showed lower activity at Emx1-1 (mean 27.7\%) with $\mathrm{C}$ C context, but highest activity at the third $\mathrm{C}$ (CCC) at Fancf (mean 39.7\%) and Emx1-3 (mean 58.0\%) (Fig. 2b-d). YE BEs effectively narrow the editing window to 1-2 nucleotides (mainly from C5 to C6) with similar or lower editing efficiency (Fig. 2b-d), consistent with previous study [7]. The eA3A-BE exhibited obvious preference for TC context, while it reduced base editing activity (Fig. 2b-d); it may be due to the fact that these three target sites were not classical TCR motifs [9]. However, no significantly narrowed editing window were observed using 7aa-BE (Fig. 2b-d), which is also consistent with previous reports that shortened linker did not substantially alter editing window [7, 9]. These results indicate that eA3G-BE system is efficient and precise in human cells with distinct $\mathrm{CC}$ context-specificity. In conjunction with previous precise BEs, eA3G-BE system could further enrich the tool kits for accurate gene editing and therapy.

\section{eA3G-BE can induce accurate C-to-T conversions in rabbit embryos}

To further evaluate the precision of eA3G-BE, six target sites including $\mathrm{CC}$ and $\mathrm{CCC}$ contexts were selected in rabbit embryos (Fig. 3a). Base editing was conducted in rabbit zygotes using microinjection of eA3G-encoding mRNA and single guide RNA (sgRNA). The control group, rA1-BE, showed a large editing window mainly from $C 3$ to $C 9(\sim 7 \mathrm{nt})$ and even induced the widest range of mutations spanning from $\mathrm{C} 2$ to $\mathrm{C} 15$ at Tyr-3 without obvious context-specificity (Fig. 3b, c). In contrast, eA3G-BE exhibited ideal efficiency comparable to that of $\mathrm{rA1}-\mathrm{BE}$ at all six sites (average editing frequencies $54.5-92.2 \%$ vs. 76.6-95.2\%) and significantly reduced bystander activities in non-CC contexts with a similar $\sim 7$ nt editing window (mainly from $\mathrm{C} 4$ to $\mathrm{C} 10$ ) 


\begin{tabular}{|c|c|c|}
\hline Target site (mutation) & Phenotype & Sequence(5'-3') \\
\hline Tia1 (p.P362L) & ALS & CAGCCGCCTCCTGGCCAGAACGG \\
\hline Tyr-1 (p.Q48Stop) & OCA1 & TGTGGCCAGCTCTCAGGCAGAGG \\
\hline Dmd (p.Q869Stop) & DMD & AAGCCAGTTAAAAATGTGTAAGG \\
\hline$T y r-2(N A)$ & NA & AGTCCCTGTGGCCAGCTCTCAGG \\
\hline Fgf5 (NA) & $N A$ & GTCCGCCCCCGCGGCTTCTCTGG \\
\hline$T y r-3(N A)$ & NA & GCTCCCCTCCTCATCAGATGTGG \\
\hline
\end{tabular}
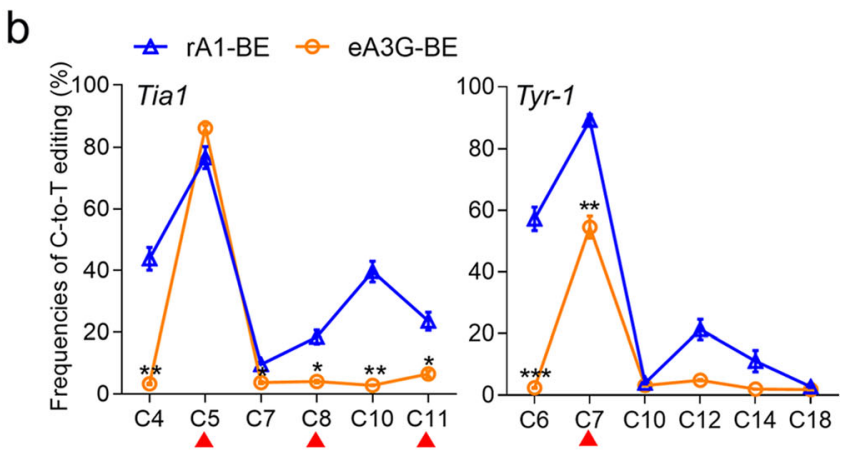

C
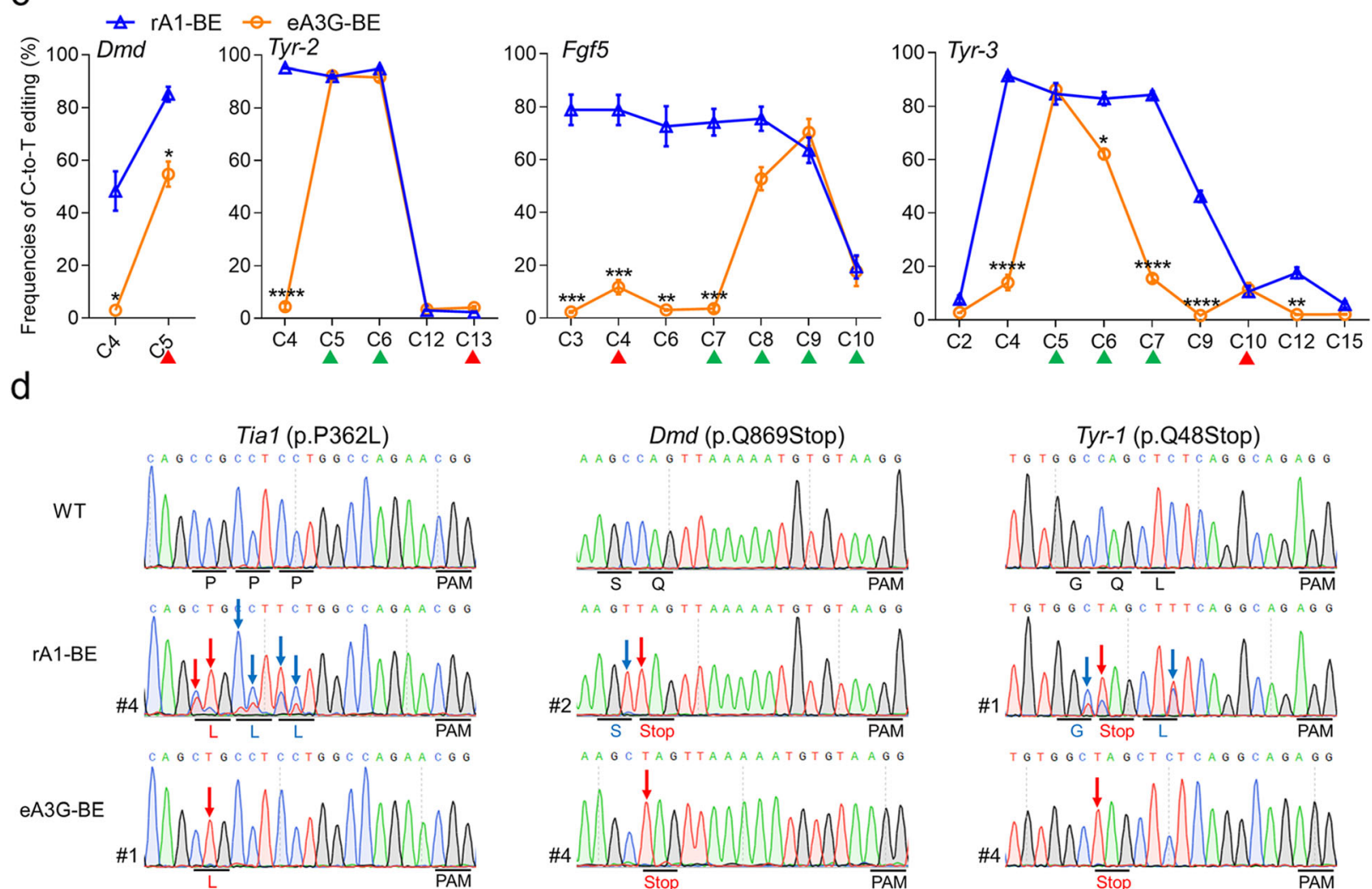

Fig. 3 eA3G-BE can induce efficient C-to-T conversions with minimized bystander activity in rabbit embryos. a The six target-site sequences with CC context. Target sequence (black), PAM region (green), target base (red), and mutant amino acid (underlined). NA, not applicable. b, c Frequencies of single C-to-T conversions using rA1-BE and eA3G-BE at six sites in rabbit embryos. CC context (red triangle) and CCC context (green triangle). Target Cs are counting the PAM as positions 21-23. $n=\sim 6$ blastocysts. $\mathbf{d}$ Representative seque ncing chromatograms of edited rabbit blastocyst at three target sites using rA1-BE and eA3G-BE systems. Targeted mutations (red arrows) and bystander mutations (blue arrows). The relevant codon identities at the target site are presented under the DNA sequence

(Fig. 3b, c). Moreover, the eA3G-BE exclusively edited the second $\mathrm{C}$ when the $\mathrm{CC}$ dinucleotide presents in the editing window, while with reduced efficiency at two $(54.7 \pm 10.7 \%$ vs. $85.1 \pm 6.3 \%, P<0.05$ at $D m d ; 54.5 \pm$ $8.8 \%$ vs. $89.3 \pm 4.2 \%, P<0.01)$ of three tested sites compared with rA1-BE (Fig. 3b, c), consistent with that in human cells. In addition, at three tested sites with multiple Cs, eA3G-BE induced high editing frequencies in CCC (Tyr-2 and Tyr-3) or CCC (Fgf5) contexts, meanwhile significantly decreased bystander activities at the first cytidine (Fig. 3c). Notably, with the high precision of eA3G-BE, targeted C-to-T conversions can be induced at target $\mathrm{C}$ without generating bystander mutations at Tia1, Dmd, and Tyr-1, thus precisely mimicking the p.P362L missense mutation of human amyotrophic lateral sclerosis (ALS) [18], the p.Q869Stop nonsense mutation of Duchenne muscular dystrophy (DMD) [19], and the p.Q48Stop nonsense mutation of oculocutaneous albinism type 1 (OCA1) [20], respectively (Fig. 3d). Overall, these results demonstrated that engineered 
eA3G-BE system can efficiently and precisely induce Cto- $\mathrm{T}$ editing in rabbit embryos with sequence preference for CC, suggesting its potential to develop animal models for precisely mimicking human genetic diseases.

\section{Precise base conversion at Tyr to recapitulate human albinism using eA3G-BE}

Subsequently, we further explored the use of eA3G-BE to generate Founder (F0) mutant rabbits. A single C-to$\mathrm{T}$ conversion was designed at Tyr-1 (p.Q48stop) to mimic human OCA1 in F0 rabbits (Fig. 4a). Rabbit zygotes were transplanted into surrogate mothers after microinjection and six pups were obtained. The result of T-A cloning showed that five of six pups (83\%) were mutants with editing frequencies from 40 to $100 \%$ (Fig. 4b and Table 1). Notably, targeted base editing at C7 was successfully induced in all of five mutants without any bystander mutations, enabling generation of highly precise p.Q48Stop mutation of OCA1 (Fig. 4b-e). Moreover, three homozygous mutants (T1-1, T1-2, and T1-3) exhibited a complete albino phenotype, and the chimeric mutants (T1-4 and T1-5) exhibited mosaic distribution of black and white skin and hair, which is consistent with their mutant genotype (Fig. 4f). Furthermore, histological H\&E staining also revealed the local or complete absence of melanin in the skin of representative T1-5 or T1-1 mutants, but not in the WT rabbit (Fig. 4g). In addition, no apparent off-target mutations were detected at potential off-target sites (POTs) in mutant rabbits, consistent with the results in human cells (Additional file 1: Fig. S5a). Thus, this rabbit model has recapitulated human OCA1 disease symptoms, underscoring the advantageous potentiality of the eA3G-BE system in precisely generating point mutation disease rabbit models.

\section{Expanded targeting scope using eA3G-NG}

The NGG PAM requirement of SpCas9 substantially limits the target sites suitable for eA3G-nCas9 fusions. Therefore, we explore the feasibility of SpCas9-NG system with currently the most relaxed NGN PAMs to expand the genome-targeting scope [21] (Fig. 5a). First, eight target sites with all NGN PAMs were selected to be tested in human cells. Notably, compared with rA1NG system, eA3G-NG showed comparable efficiency and distinct preference for $\mathrm{CC}$ context in all tested sites (Fig. 5b and Additional file 1: Fig. S6). Subsequently, three target sites with NGT or NGA PAM which are arduous to edit using conventional SpCas9-BE were selected to be tested in rabbit embryos (Fig. 5c). Notably, eA3G-NG exhibited high target efficiency comparable to that of rA1-NG at all three sites while significantly reduced bystander activities in non-CCC contexts, which, in turn, substantially decreased unwanted bystander amino acid mutations (Fig. 5d). In particular, with both high precision and expanded space of eA3G-NG, accurate p.P301L mutation can be induced in Mapt gene to precisely mimic human classical p.P301L missense mutation of Alzheimer's disease (AD) [22]. It is extremely difficult for conventional rA1-NG to induce desired p.P301L mutation as it only induces undesired p.P301F mutation due to its high frequencies of bystander C-toT editing (Fig. 5e).

Furthermore, the optimized eA3G-NG was used to generate F0 rabbits that carry the Tyr-4 (p.W218Stop) mutation in order to mimic human OCA1 (Fig. 6a). Five pups were obtained and all of them (100\%) were homozygous with desired nonsense mutation, consistent with the high efficiency in embryos (Fig. $6 \mathrm{~b}$ and Table 1). Strikingly, no obvious bystander mutations were observed in all rabbits (Fig. 6b-e). All five pups (100\%) showed a systemic albino phenotype (Fig. 6f). Moreover, histological H\&E staining revealed the absence of melanin in the skin of representative T4-3, but not in the WT rabbit (Fig. 6g). No obvious off-target mutations were detectable at POTs in mutant rabbits (Additional file 1: Fig. S5b). These results indicated the eA3G-NG is highly efficient at relaxed NG PAMs in rabbit and possesses excellent prospects for precisely mimicking human pathogenic point mutations in animal models.

\section{Loop3 and Loop7 jointly determine the CC context- specificity of eA3G-BE}

It is well known that hA3G is the only cytidine deaminase family member with a unique intrinsic preference for $C C$ dinucleotides in vitro $[12,13]$. Our results also confirmed that eA3G-BE maintains a strong CC context-specificity, and we want to explore what determines its unique preference for CC context. In previous reports, hA3A had an intrinsic preference for cytosine preceded by thymine (TC context) in vitro which is different from hA3G $[9,23]$. To better understand the differential dinucleotide context-specificity of hA3G and hA3A, we aligned the amino acid sequences of the hA3A and catalytic domain of hA3G (residues 197-384) (Fig. 7a). We found that the protein sequence of hA3A is highly homologous with that of hA3G-CTD, but it is quite different in two key DNA binding loops, loop3 and loop7 (Fig. 7a). In addition, previous study has shown that the loop 3 influences enzymatic activity and loop 7 alone governs the intrinsic preference for $\mathrm{CC}$ dinucleotides [24].

Here, to further determine the key loop area responsible for CC context-specificity of eA3G-BE, we constructed a series of chimeras systems, termed eA3GL3A, eA3G-L7A, and eA3G-L3L7A, in which loop 3 and loop 7 of the hA3G-CTD were replaced with the corresponding loop regions from hA3A (Fig. 7b). Three target 


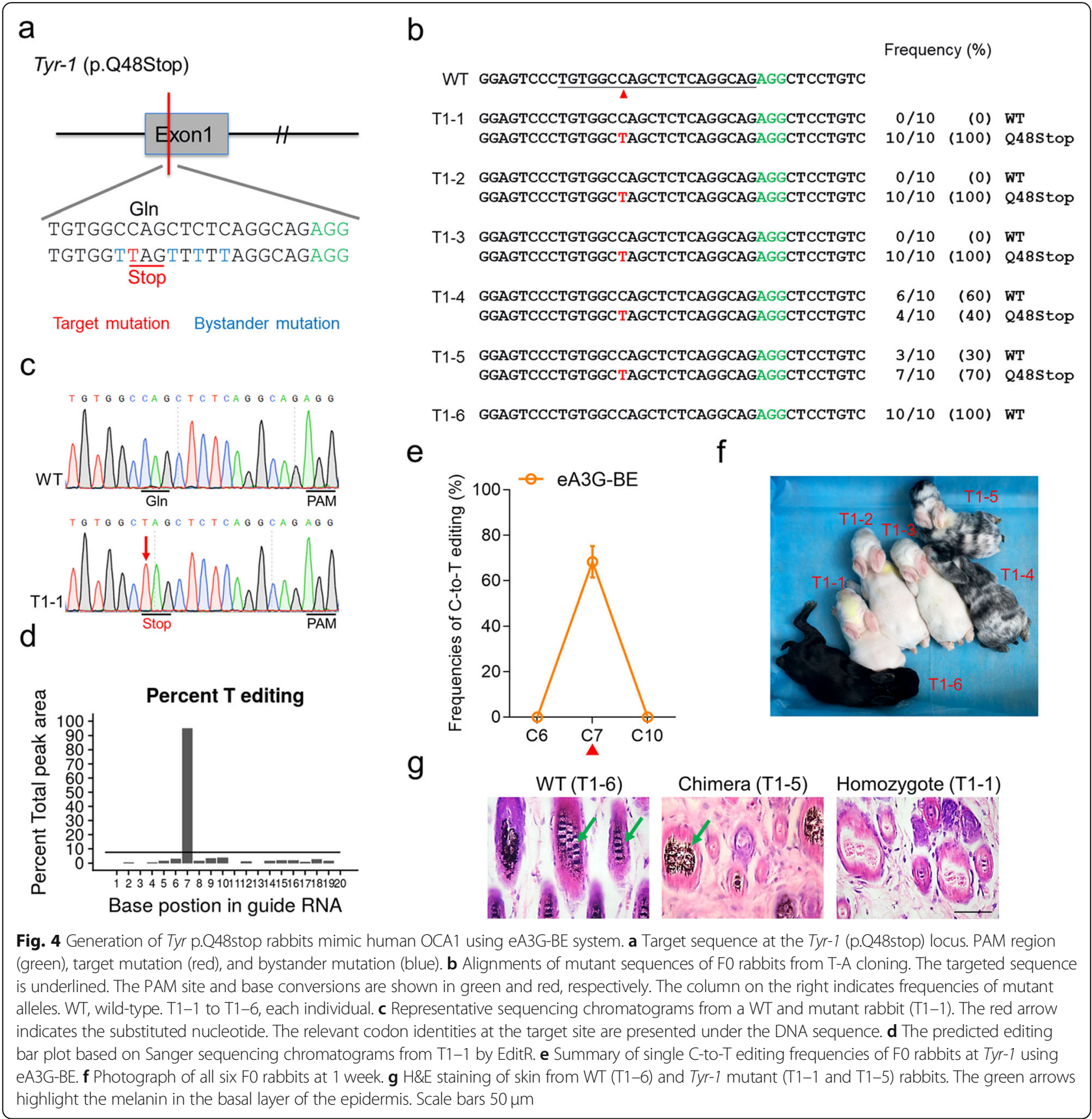

Table 1 Summary of F0 rabbits generated in this study

\begin{tabular}{lllllll}
\hline \multirow{2}{*}{ System } & & & & \multicolumn{2}{l}{ Mutant ratio (\%) } & \\
\cline { 6 - 7 } \cline { 5 - 7 } & Target site & Embryos transferred & No. of offspring & No. of mutants & No. of homozygous mutants & No. of bystander mutants \\
\hline eA3G-BE & Tyr-1 p.Q48Stop & 42 & 6 & $5(83)$ & $3(50)$ & $0(0)$ \\
eA3G-NG & Tyr-4 p.W218Stop & 38 & 5 & $5(100)$ & $5(100)$ & $0(0)$ \\
\hline
\end{tabular}



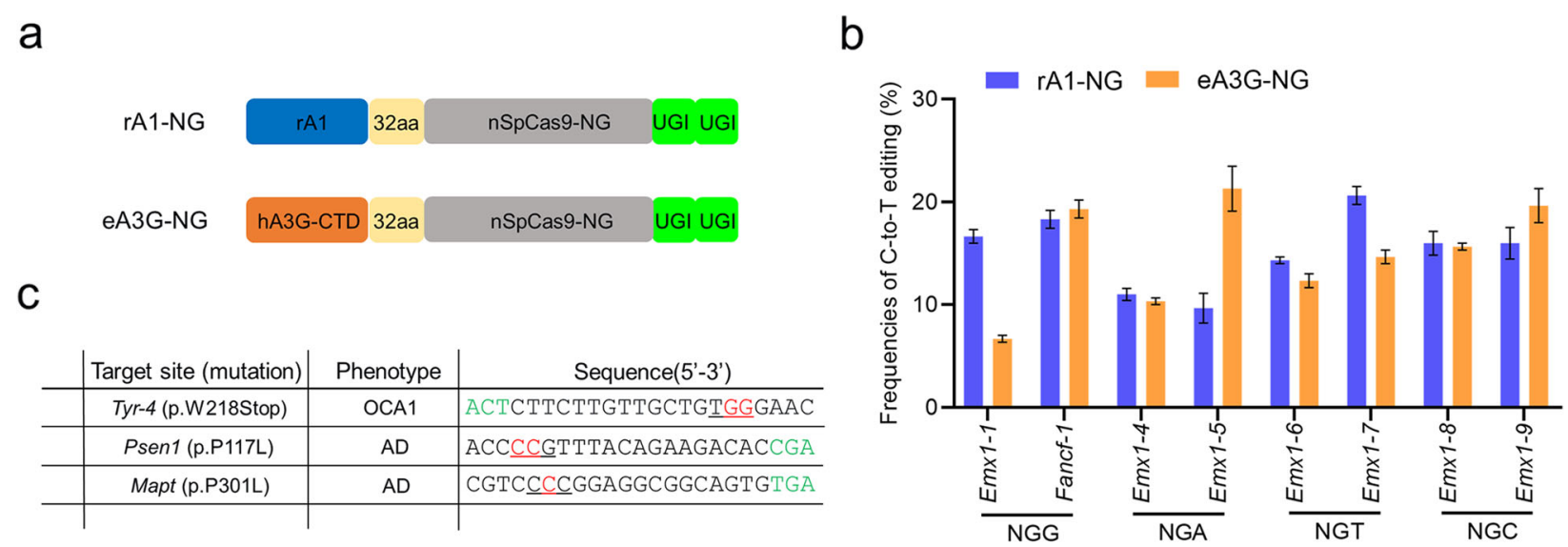

d

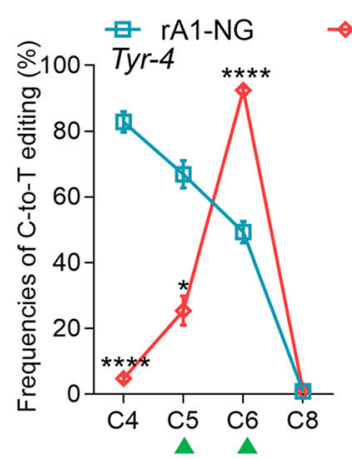

\section{eA3G-NG}
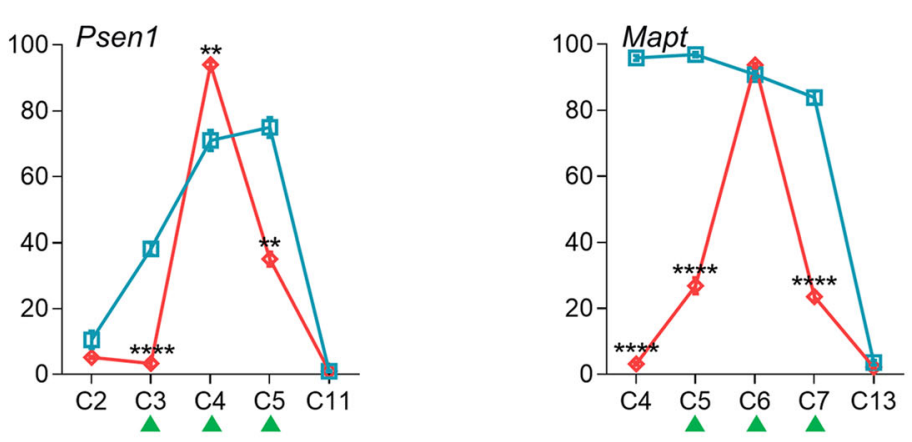

e
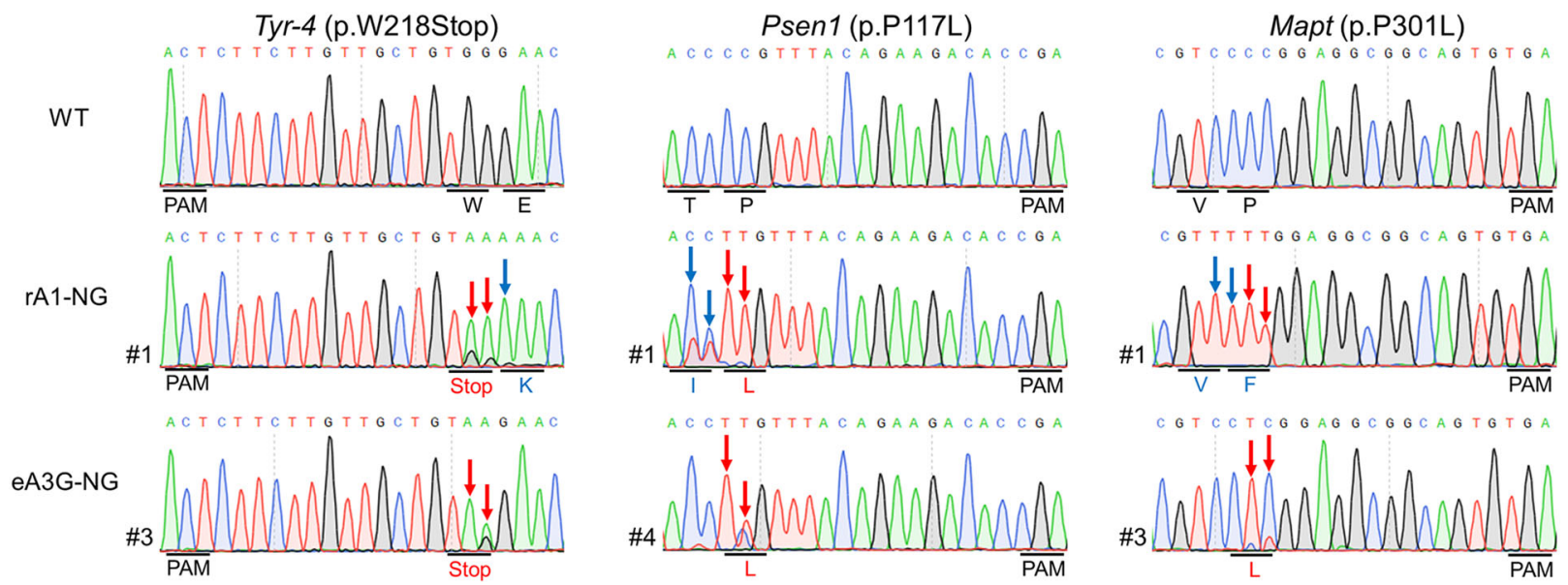

Fig. 5 Expanded targeting scope using optimized eA3G-NG fusion in rabbit embryos. a Schematic representation of rA1-NG and eA3G-NG architecture. b Comparison of the base editing frequencies between rA1-NG and eA3G-NG with NGN PAMs in HEK293T cells. Values and error bars reflect the mean \pm s.e.m. of three independent biological replicates. c The three target-site sequences within NG PAMs. Target sequence (black), PAM region (green), target base (red), and mutant amino acid (underlined). $\mathbf{d}$ Comparison of the editing frequencies of single C-to-T conversions between rA1-NG and eA3GNG at three sites with NG PAMs in rabbit embryos. CCC context (green triangle). $n=\sim 6$ blastocysts. e Representative sequencing chromatograms of edited rabbit blastocyst at three target sites using rA $\overline{1-N G}$ and eA3G-NG systems. Targeted mutations (red arrows) and bystander mutations (blue arrows). The relevant codon identities at the target site are presented under the DNA sequence

sites were tested to directly compare the editing activities of five base editor fusions: the original $\mathrm{rA1}-\mathrm{BE}$, $\mathrm{A} 3 \mathrm{~A}-\mathrm{BE}$, and eA3G-BE and three eA3G-BE variants, eA3G-L3A, eA3G-L7A, and eA3G-L3L7A. Consistent with previous results, the $\mathrm{A} 3 \mathrm{~A}-\mathrm{BE}$ induce similar efficiency compared with that of $\mathrm{rA1}-\mathrm{BE}$ while showed higher editing activity in a GC context and a larger editing window $[10,15]$ (Fig. $7 \mathrm{c}-\mathrm{e}$ and Additional file 1: Fig. S7). It was unexpected that both eA3G-L3A and eA3GL7A lost original CC context-specificity compared with 


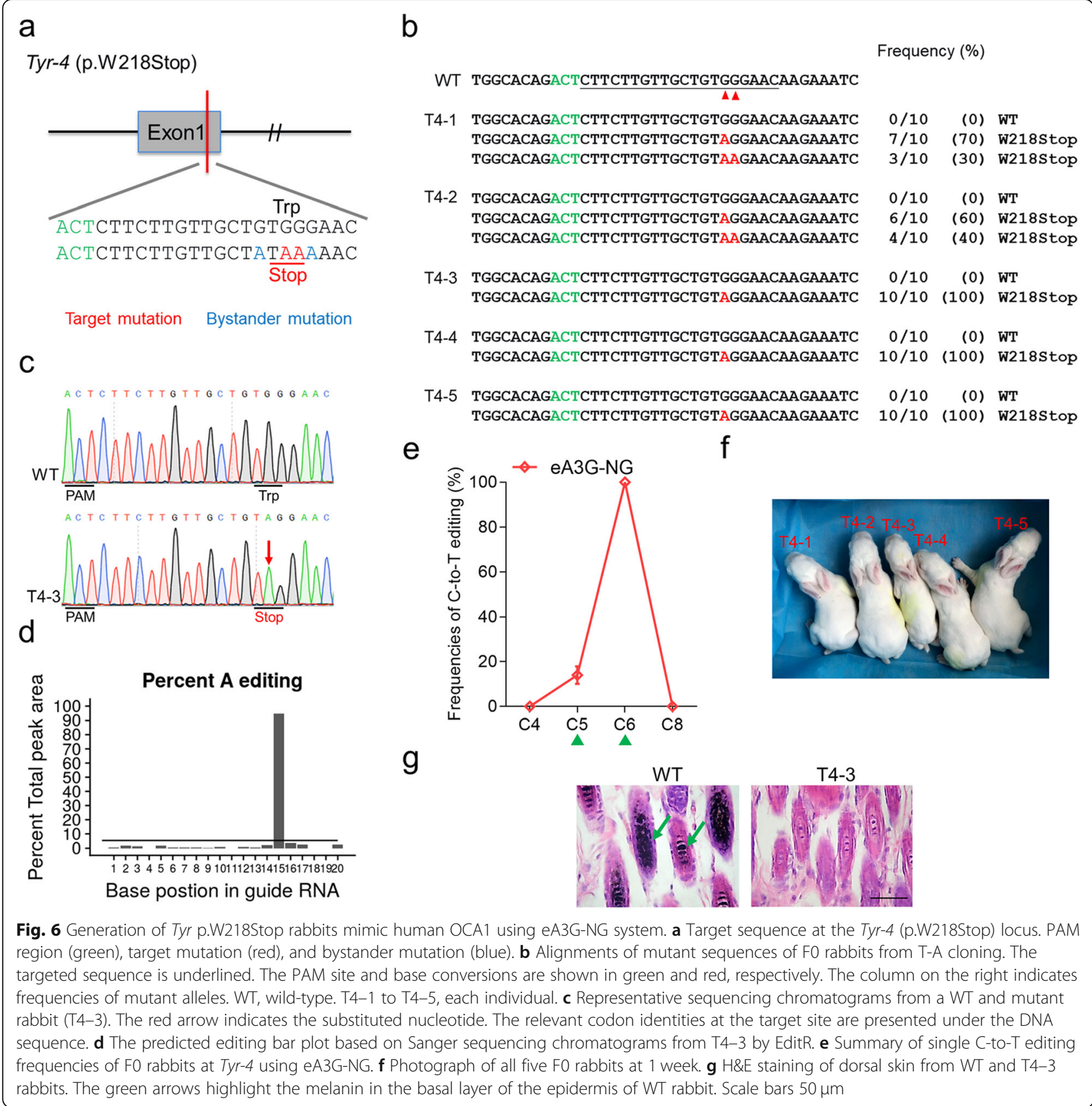

eA3G-BE in all three tested sites, while with similar or lower overall activity (Fig. 7c-e). In particular, the eA3G-L7A apparently changed the context preference from $\mathrm{CC}$ to TC (Fig. 7c-e). Furthermore, the eA3GL3L7A, which combines both eA3G-L3A and eA3GL7A, exhibited high editing efficiency, compatibility of GC context, and a large editing window, similar to that of A3A-BE (Fig. 7c-e). Due to its larger editing window and independently of context, the new developed eA3GL3L7A could initiate base conversions more extensively and increase genome-targeting scope, including the induction of stop (iSTOP) codons and mutation of multiple sites within the gene regulatory regions [25, 26]. Taken together, these results showed that both loop3 and loop7 jointly govern the CC contextspecificity of eA3G-BE and simultaneous replacement of loop 3 and loop 7 resulted in a hyperactive eA3G-L3L7A variant similar to $\mathrm{A} 3 \mathrm{~A}-\mathrm{BE}$, suggesting the potential of developing new and versatile base editors by grafting key loop region.

\section{Discussion}

The base editing precision represents a primary obstacle for base editors, especially for precise disease modeling 


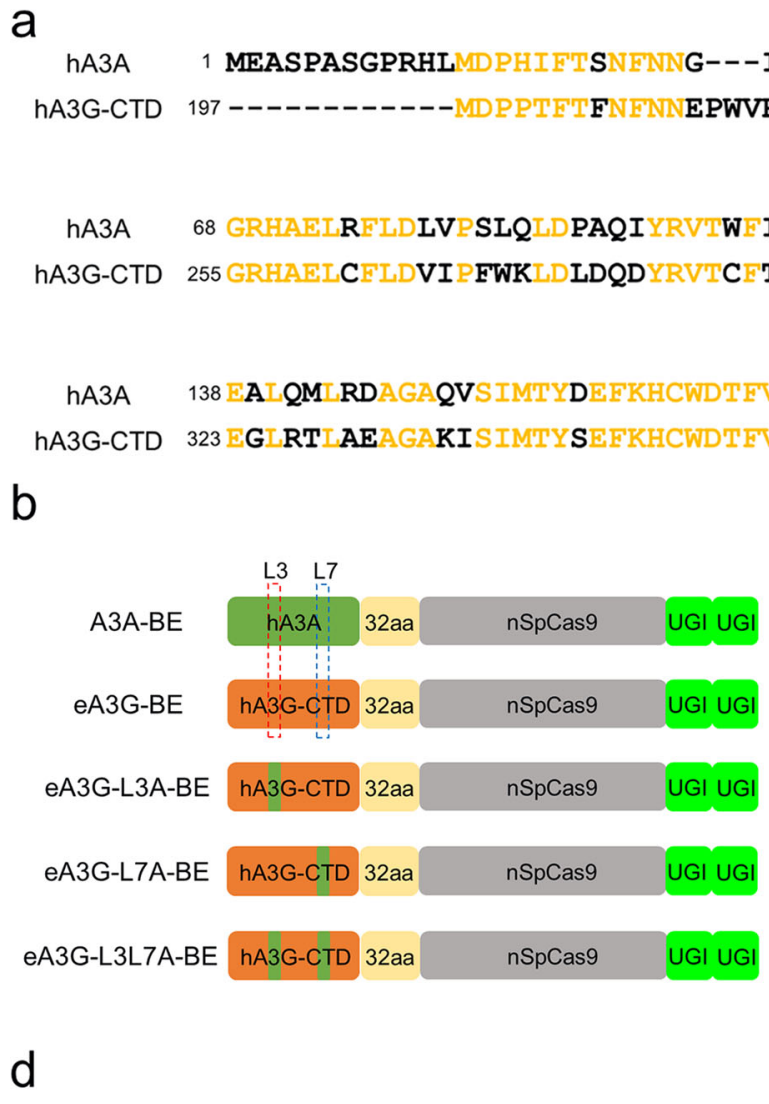

a

b

$\llcorner 3 \quad\llcorner 7$

$\begin{array}{rrrrr}\text { A3A-BE } & \text { hA3A } & \text { 32aa } & \text { nSpCas9 } & \text { UGI UGI } \\ \text { AA3G-BE } & \text { hA3G-CTD 32aa } & \text { nSpCas9 } & \text { UGI UGI } \\ \text { eA3G-L3A-BE } & \text { hA3G-CTD 32aa } & \text { nSpCas9 } & \text { UGI UGI } \\ \text { eA3G-L7A-BE } & \text { hA3G-CTD 32aa } & \text { nSpCas9 } & \text { UGI UGI } \\ \text { eA3G-L3L7A-BE hA3G-CTD 32aa } & \text { nSpCas9 } & \text { UGI UGI } \\ \text { d } & & & \\ & & & \end{array}$
hA3A 1 MEASPASGPRHL PHIFT SNENIG---IGRHTYLCYEVERLD G S KMD HRGELHNQ KNLLCGFY 67

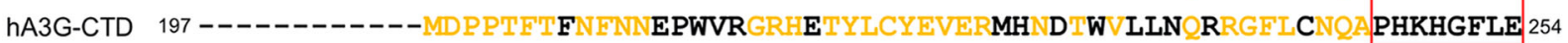
\begin{tabular}{l} 
Loop 3 \\
\hline
\end{tabular}

hA3A 68 GRHAEIR LDIV SLQLDAQIYRVIW I SWSPCFSW CA G VRA LQE THVR RIFARIYDYDPLYK 137 hA3G-CTD 255 GRHAELCELDI FWK IDQDYRVTCTSWPCFS--CAQ MAK ISK K SICIF TARIYDDQGRCQ 322

Loop 7

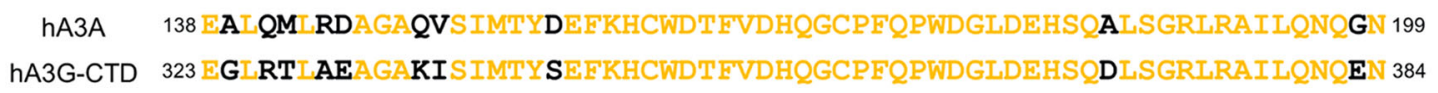

Fancf-1: GGAATC $\mathrm{C}_{7} \mathrm{C}_{8} \mathrm{TTC}_{11} \mathrm{TGC}_{14} \mathrm{AGCACCTGG}$

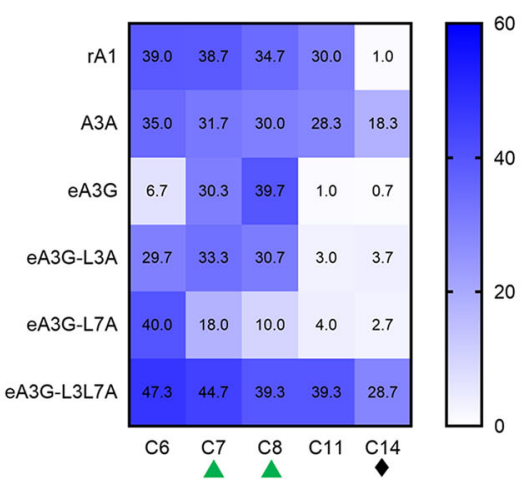

C

Emx1-1: GAGTC $_{5} \mathrm{C}_{6} \mathrm{GAGC}_{10} \mathrm{AGAAGAAGAAGGG}$

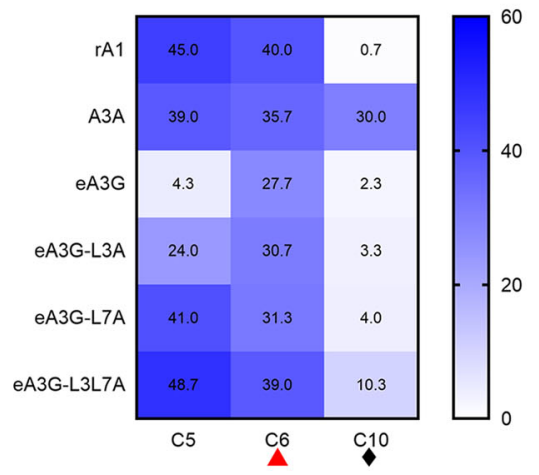

e

Emx1-3: $\mathrm{TGC}_{3} \mathrm{C}_{4} \mathrm{C}_{5} \mathrm{C}_{6} \mathrm{TC}_{8} \mathrm{C}_{9} \mathrm{C}_{10} \mathrm{TC}_{12} \mathrm{C}_{13} \mathrm{C}_{14}$ TGGCCCAGG

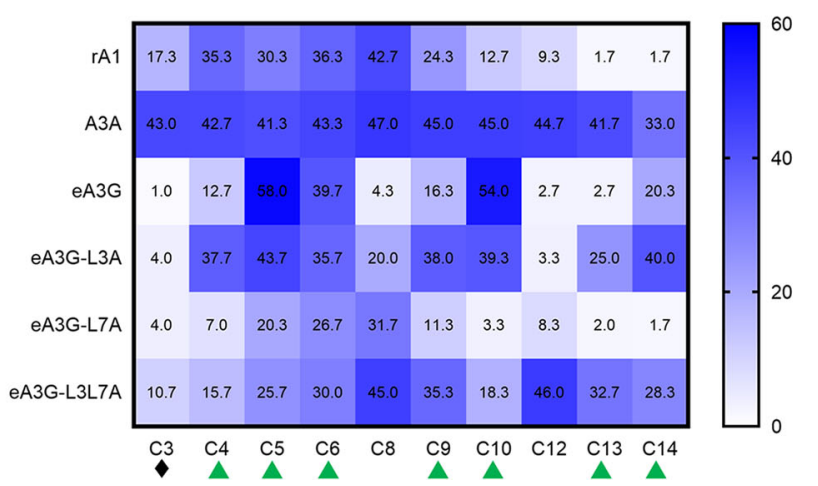

Fig. 7 Comparison of base editing activity using loop3 and loop 7 grafted eA3G-BEs. a Amino acid alignments of hA3A and hA3G-CTD. The loop 3 and 7 regions are shown in red and blue rectangular box, respectively. $\mathbf{b}$ Schematic representation of five base editors' architecture. The dotted rectangle box indicates loop 3 and loop7 region. c-e Heat maps showing C-to-T editing efficiencies for six base editors at Emx1-1 (c), Fancf (d), and Emx1-3 (e). The CC or CCC context was shown in red triangle or green triangle, respectively. The GC context was shown in black diamond. Values and error bars reflect the mean \pm s.e.m. of three independent biological replicates

and gene therapy. Here, we attempted to engineer a fusion of base editors with hA3G, which successfully improved the precision of eA3G-BE with the distinctive preference for CC context. Targeted editing efficiency of 18.3-58.0\% or $54.5-92.2 \%$ with excellent CC contextspecificity was observed in human cells or rabbit embryos, respectively. In addition, by fusing the eA3GBE with SpCas9-NG, the eA3G-NG with relaxed NG PAMs notably expanded the genome-targeting scope. Moreover, eA3G-BEs were used to induce site-specific single-base substitutions with $83-100 \%$ efficiency and few bystander mutations in F0 rabbits at Tyr-1 
p.Q48stop and Tyr-4 p.W218Stop loci. These data indicated that the efficient and precise eA3G-BEs can be used as a reliable tool for inducing high-precision base editing in rabbits.

Moreover, in this study, we found that both loop 3 and loop 7 jointly determine the CC context-specificity of eA3G-BE. It is worth noting that the loop 3 replacement only enhanced hA3G catalytic activity but did not alter its intrinsic $5^{\prime}$-CC dinucleotide substrate preference in vitro [24]. This divergence may be due to the difference of in vitro and in vivo experimental environment and the influence of the architecture of cytidine deaminase-Cas9 fusion [9]. Taking advantage of deaminase's natural preference and appropriate artificial design may be a novel perspective to develop new base editing tools. During our preparation of this manuscript, a similar study of engineered APOBEC3G-nCas9 base editors was reported [27]. The editing efficiency in CC context of A3G-BEs was improved through rational engineering A3G deaminase [27]. Therefore, it may be an effective strategy to enhance $\mathrm{CC}$ motif preference and increase the editing efficiency of eA3G-BE by changing the key residues.

Additionally, a series of base editors with high precision were developed by narrowing activity window of deaminases, such as YE-rA1 [7], YFE-rA1 [28], truncated CDA1 [8], truncated A3A [29], and rationally designed A3B [30]. Context-dependent base editors, including eA3A-BEs [9] and eA3G-BEs, represent an important advance that offers more precise base editing, while they may lower the target site applicability because the target nucleotide must naturally exist in the preferred sequence context [6]. Therefore, selecting reasonable base editors based on their specific features, such as narrowed window and contextspecificity, to preferentially edit the target base over the bystander base so as to obtain desired editing results for the specific sites could be the future trend.

In addition, the requirement of NG PAM still limits the number of target sites suitable for eA3G-BEs. To date, numerous SpCas9 homologs and variants that recognize a variety of PAMs have been found, such as SaCas9 (NNGRRT) [31], Cpf1 (TTTV) [32], NmeCas9 (N4GATT) [33], ScCas9 (NNG) [34], Cas12b (TTN) [35, 36], and Nme2Cas9 (N4CC) [37]. They may further improve the genome-targeting scope of eA3G-BE system when combined with it.

It has been showed that base editors may cause genome-wide off-target DNA and RNA mutations [3841]. These unexpected off-target DNA and RNA mutations are mainly caused by deaminase domain rather than Cas9 domain. Additionally, the off-target DNA and RNA editing could be eliminated by rational mutagenesis of deaminase domain [42-44]. It has been demonstrated that the Cas9-independent off-targets of hA3G-
$\mathrm{BE}$ is relatively low due to its $\mathrm{CC}$ context-specificity [42]. However, more detailed and diverse examination is required to evaluate eA3G-BEs in future investigation.

The eA3G-BEs can precisely correct point mutations without requiring a DNA-repair template, which makes them as promising tools in gene therapy. However, eA3G-BEs cannot be packaged in a single adeno associated virus (AAV) vector due to AAV packaging limit of $\sim 4.7 \mathrm{kbp}$. It may be solved by using a dual trans-splicing adeno-associated virus (tsAAV) vector system or a splitintein base editor to circumvent the limited cargo capacity of AAV vectors, which have been successfully used to treat many genetic diseases, such as ALS and neurodegenerative ataxia [45-48]. Additionally, finding or designing smaller variants of Cas9 and deaminase is also an effective approach in the future.

\section{Conclusions}

In summary, we develop a series of eA3G-nCas9 fusions that can induce efficient base editing with minimized bystander activity in CC motifs. The eA3G-BE can function as a generic version of the $\mathrm{CC}$ context-dependent base editor, and the engineered eA3G-NG further expands genome-targeting scope with relaxed NG PAMs. Thus, these eA3G-nCas9 base editors improve the precision and expand the scope of the currently used rA1-BEs system and have a great potential to be promising tools for precise animal model establishment and gene therapy in the future.

\section{Methods}

\section{Plasmid construction}

The rA1-BE4max was obtained from Addgene (\#112093). The DNA fragment of hA3G-CTD, eA3G-L3A, eA3G-L7A, and eA3G-L3L7A was synthesized and cloned into rA1BE4max by Genscript Biotech (Nanjing). Seven mutations (R1335A/L1111R/D1135V/G1218R/E1219F/A1322R/T1337

R) of SpCas9 were introduced into rA1-BE4max and eA3GBE4max to create rA1-NG and eA3G-NG. Plasmid sitedirected mutagenesis was performed using the Fast Site-Directed Mutagenesis Kit (TIANGEN, Beijing). All the site-directed mutation primers are listed in Additional file 1: Table S1. The amino acid sequences of plasmids are listed in Additional file 1: Supplementary sequence.

\section{Design guidelines of gRNA for eA3G-BEs}

The first step in gRNA design is to identify available PAMs (NGG of eA3G-BE and NGN of eA3G-NG) that would place the target $C$ within the major editing window (positions 4-10 in the gRNA target site).

For two-C motifs, eA3G-BEs edit the second C $(\mathrm{C} \underline{\mathrm{C}})$ with similar or lower editing efficiency (site-dependent) compared with rA1-BEs. For three-C motifs, compared 
with rA1-BEs, eA3G-BEs edit the second C (CCC) with similar or lower editing efficiency (site-dependent) and edit the third C (CCC) with comparable editing efficiency. For motifs containing more than three Cs, eA3G-BEs edit the fourth or more Cs (CCCC) with similar or reduced editing efficiency (site-dependent) compared with rA1-BEs.

\section{Cell culture and transfection}

Human kidney epithelial cell line (HEK293T) were cultured in Dulbecco's modified Eagle's medium (DMEM) supplemented with $10 \%$ fetal bovine serum (HyClone) and incubated at $37^{\circ} \mathrm{C}$ in an atmosphere of $5 \% \mathrm{CO}_{2}$. The cells were seeded into 6-well plates and transfected using Lipofectamine ${ }^{\text {Tm }} 3000$ Reagent (Thermo Fisher Scientific) according to the manufacturer's instructions. After $72 \mathrm{~h}$, the cells were collected and used for genotyping. All primers used for genotyping are listed in Additional file 1: Table S2.

\section{mRNA and gRNA preparation}

All plasmids were linearized with NotI and transcribed in vitro using the HiScribe ${ }^{\mathrm{ma}}$ T7 ARCA mRNA kit (NEB). mRNA was purified using the RNeasy Mini Kit (Qiagen) according to the manufacturer's protocol. The sgRNA oligos were annealed into pUC57-sgRNA expression vectors containing a $\mathrm{T} 7$ promoter. The sgRNAs were then amplified and transcribed in vitro using the MAXIscript T7 kit (Ambion) and purified using the miRNeasy Mini Kit (Qiagen) according to the manufacturer's protocol.

\section{Microinjection of rabbit zygotes}

The protocol used for the microinjection of pronuclearstage embryos has been described in detail in our previously published study [49]. Briefly, a mixture of mRNA $(200 \mathrm{ng} / \mu \mathrm{l})$ and sgRNA (50 ng/ $\mu \mathrm{l})$ was co-injected into the cytoplasm of pronuclear-stage zygotes. The injected embryos were transferred into EBSS medium for shortterm culture at $38.5{ }^{\circ} \mathrm{C}, 5 \%$ carbon dioxide, and $100 \%$ humidity. Then, approximately 30-50 injected zygotes were transferred into the oviducts of recipient rabbit.

\section{Single-embryo PCR amplification and rabbit genotyping}

Each group was injected with an average of approximately 10 embryos to test the base editing efficiency. The injected embryos were transferred into EBSS medium for culture at $38.5{ }^{\circ} \mathrm{C}, 5 \%$ carbon dioxide, and $100 \%$ humidity. Then, the injected embryos were collected at the blastocyst stage. Genomic DNA was extracted in embryo lysis buffer (1\% NP40) at $56^{\circ} \mathrm{C}$ for 60 min, then at $95^{\circ} \mathrm{C}$ for 10 min in a BIO-RAD PCR Amplifier. Then, the extracted products were amplified by PCR $\left(95^{\circ} \mathrm{C}, 5 \mathrm{~min}\right.$ for pre-degeneration, 42 cycles of $\left(95^{\circ} \mathrm{C}, 30 \mathrm{~s}, 58^{\circ} \mathrm{C}, 30 \mathrm{~s}, 72{ }^{\circ} \mathrm{C}, 30 \mathrm{~s}\right), 72^{\circ} \mathrm{C}, 5 \mathrm{~min}$ for extension) and determined by Sanger sequencing. The Sanger sequencing result of each blastocyst was used to evaluate base editing frequencies by EditR [17]. The genomic DNA of newborn rabbits was extracted from ear clips and analyzed by PCR genotyping, Sanger sequencing, and T-A cloning. All primers used for genotyping are listed in Additional file 1: Table S2.

\section{Off-target assay}

The top five potential off-target sites for each gRNA were predicted to analyze site-specific edits according to Cas-OFFinder [50] (http://www.rgenome.net/cas-offinder/). All primers for the off-target assay are listed in Additional file 2: Table S1.

\section{Hematoxylin and eosin (H\&E) staining}

The dorsal skin from WT and mutant rabbits was fixed in $4 \%$ paraformaldehyde for $48 \mathrm{~h}$, embedded in paraffin wax, and then sectioned for slides. Slides were stained with $H \& E$ and viewed under a Nikon ts100 microscope.

\section{Western blotting}

Western blotting analyses were performed, as described previously [51]. The samples from the transfected HEK293T cells were lysed in RIPA buffer supplemented with a protease inhibitor cocktail (Roche, Basel, Switzerland). The antibody against Cas9 (1:1500; ab204448, Abcam) was used as a primary antibody, while tubulin antibody (1:2000; 10094-1-AP, Wuhan Sanying) was used as the loading control.

\section{Statistical analysis}

All data are expressed as mean \pm s.e.m. of at least three individual determinations for all experiments. Data were analyzed by Student's $t$ test via GraphPad Prism software 8.0.1. The probability value smaller than $0.05(p<0.05)$ is considered to be statistically significant. ${ }^{*} p<0.05$, ${ }^{* * *} p<0.01,{ }^{* * * *} p<0.001,{ }^{* * * * * *} p<0.0001$.

\section{Supplementary information}

Supplementary information accompanies this paper at https://doi.org/10. 1186/s12915-020-00849-6.

\footnotetext{
Additional file 1: Figure S1. Immunoblots of the rA1-BEs and eA3GBEs. Figure S2. Comparison of base editing frequencies in non-CC contexts with rA1-BE and eA3G-BE. Figure S3. Comparison of base editing frequencies at on- and off-target sites with $r A 1-B E$ and eA3G-BE. Figure S4. Comparison of base editing activity and precision using eA3G-BE and other precise BEs. Figure S5. Representative sequencing chromatograms of off-target detection in Tyr-1 and Tyr-4 mutant rabbits. Figure S6. Comparison of base editing frequencies between rA1-NG and eA3G-NG at 8 target sites with all NGN PAMs. Figure S7. Comparison of base editing activity using loop3 and loop 7 grafted eA3G-BEs. Table S1. Primers used for site-directed mutation in this study. Table S2. Primers used for genotyping in this study. Supplementary sequence. Amino acid sequence of eA3G-BE.
} 
Additional file 2: Table S1. The primers used for identifying potential off-target sites in this study.

\section{Abbreviations}

CBEs: Cytidine base editors; nCas9: Cas9 nickase; eA3G: Engineered human APOBEC3G; F0: Founder; CRISPR: Clustered regularly interspaced short palindromic repeat; DSBs: Double-strand breaks; indels: Insertion/deletion mutations; SpCas9: Streptococcus pyogenes Cas9; PAM: Protospacer adjacent motif; eA3A: Engineered human APOBEC3A; CTD: C-terminal catalytic domain; sgRNAs: Single guide RNAs; ALS: Amyotrophic lateral sclerosis; DMD: Duchenne muscular dystrophy; OCA1: Oculocutaneous albinism type 1; POTs: Potential off-target sites; AD: Alzheimer's disease; AAV: Adenoassociated virus

\section{Acknowledgements}

The authors thank Peiran Hu and Nannan Li for assistance at the Embryo Engineering Center for critical technical assistance.

\section{Authors' contributions}

$\mathrm{ZL}, \mathrm{LL}$, and $\mathrm{ZL}$ conceived and designed the experiments. ZL, SC, and HS performed the experiments. ZL, HS, and SC analyzed the data. YJ, MC, and YS contributed reagents/materials/analysis tools. ZL and ZL wrote the paper. All authors have read and approved the final manuscript.

\section{Funding}

This study was financially supported by the National Key Research and Development Program of China Stem Cell and Translational Research (2017YFA0105101), the Program for Changjiang Scholars and Innovative Research Team in University (No.IRT_16R32), the Strategic Priority Research Program of the Chinese Academy of Sciences (XDA16030501, XDA16030503), and Key Research \& Development Program of Guangzhou Regenerative Medicine and Health Guangdong Laboratory (2018GZR1 10104004).

\section{Availability of data and materials}

The authors state that all data necessary for confirming the conclusions presented in this article are represented fully within the article or can be provided by the authors upon request. All data generated or analyzed during this study are included in this published article and its supplementary information files.

\section{Ethics approval and consent to participate}

New Zealand white and Lianshan black rabbits were obtained from the Laboratory Animal Center of Jilin University (Changchun, China). All animal studies were conducted according to experimental practices and standards approved by the Animal Welfare and Research Ethics Committee at Jilin University.

\section{Competing interests}

The authors declare no competing interests.

\section{Author details}

'Key Laboratory of Zoonosis Research, Ministry of Education, College of Animal Science, Jilin University, Changchun 130062, China. ${ }^{2}$ CAS Key Laboratory of Regenerative Biology, Guangdong Provincial Key Laboratory of Stem Cell and Regenerative Medicine, South China Institute for Stem Cell Biology and Regenerative Medicine, Guangzhou Institutes of Biomedicine and Health, Chinese Academy of Sciences, Guangzhou 510530, China. ${ }^{3}$ Guangzhou Regenerative Medicine and Health Guang Dong Laboratory (GRMH-GDL), Guangzhou 510005, China. Institute for Stem Cell and Regeneration, Chinese Academy of Sciences, Beijing 100101, China.

\section{Received: 7 January 2020 Accepted: 16 August 2020}

\section{Published online: 31 August 2020}

\section{References}

1. Knott GJ, Doudna JA. CRISPR-Cas guides the future of genetic engineering Science. 2018:361(6405):866-9.

2. Komor AC, Kim YB, Packer MS, Zuris JA, Liu DR. Programmable editing of a target base in genomic DNA without double-stranded DNA cleavage. Nature. 2016;533(7603):420-4.
3. Gaudelli NM, Komor AC, Rees HA, Packer MS, Badran AH, Bryson DI, Liu DR. Programmable base editing of $A^{*} T$ to $G^{*} C$ in genomic DNA without DNA cleavage. Nature. 2017;551(7681):464-71.

4. Yang B, Yang L, Chen J. Development and application of base editors. Crispr J. 2019;2(2):91-104.

5. Liu Z, Chen M, Chen S, Deng J, Song Y, Lai L, Li Z. Highly efficient RNAguided base editing in rabbit. Nat Commun. 2018;9(1):2717.

6. Rees HA, Liu DR. Base editing: precision chemistry on the genome and transcriptome of living cells. Nat Rev Genet. 2018;19(12):770-88.

7. Kim YB, Komor AC, Levy JM. Increasing the genome-targeting scope and precision of base editing with engineered Cas9-cytidine deaminase fusions. Nat Biotechnol. 2017;35(4):371-6.

8. Tan J, Zhang F, Karcher D, Bock R. Engineering of high-precision base editors for site-specific single nucleotide replacement. Nat Commun. 2019; 10(1):439.

9. Gehrke JM, Cervantes O, Clement MK. An APOBEC3A-Cas9 base editor with minimized bystander and off-target activities. Nat Biotechnol. 2018;36(10): 977-82.

10. Liu Z, Chen S, Shan H, Zhang Q, Chen M, Lai L, Li Z. Efficient and precise base editing in rabbits using human APOBEC3A-nCas9 fusions. Cell Discovery. 2019;5(1):31.

11. Ziegler SJ, Liu C, Landau M, Buzovetsky O, Desimmie BA, Zhao Q, Sasaki T, Burdick RC, Pathak VK, Anderson KS, et al. Insights into DNA substrate selection by APOBEC3G from structural, biochemical, and functional studies. PLoS One. 2018;13(3):e0195048.

12. Chen KM, Harjes E, Gross PJ, Fahmy A, Lu Y, Shindo K, Harris RS, Matsuo H. Structure of the DNA deaminase domain of the HIV-1 restriction factor APOBEC3G. Nature. 2008;452(7183):116-9.

13. Holden LG, Prochnow C, Chang YP, Bransteitter R, Chelico L, Sen U, Stevens RC, Goodman MF, Chen XS. Crystal structure of the anti-viral APOBEC3G catalytic domain and functional implications. Nature. 2008;456(7218):121-4.

14. Komor AC, Zhao KT, Packer MS. Improved base excision repair inhibition and bacteriophage Mu Gam protein yields C:G-to-T:A base editors with higher efficiency and product purity. Science Advances. 2017;3(8):eaao4774.

15. Wang $X$, Li J, Wang Y, Yang B. Efficient base editing in methylated regions with a human APOBEC3A-Cas9 fusion. Nat Biotechnol. 2018;36(10):946-9.

16. Koblan LW, Doman JL, Wilson C. Improving cytidine and adenine base editors by expression optimization and ancestral reconstruction. Nat Biotechnol. 2018;36(9):843-6.

17. Kluesner MG, Nedveck DA, Lahr WS, Garbe JR, Abrahante JE, Webber BR, Moriarity BS. EditR: a method to quantify base editing from Sanger sequencing. CRISPR J. 2018;1 (3):239-50.

18. Mackenzie IR, Nicholson AM, Sarkar M, Messing J, Purice MD, Pottier C, Annu K, Baker M, Perkerson RB, Kurti A, et al. TIA1 mutations in amyotrophic lateral sclerosis and frontotemporal dementia promote phase separation and alter stress granule dynamics. Neuron. 2017;95(4):808-16 e809.

19. Mendell JR, Shilling C, Leslie ND, Flanigan KM, al-Dahhak R, Gastier-Foster J, Kneile K, Dunn DM, Duval B, Aoyagi A et al: Evidence-based path to newborn screening for Duchenne muscular dystrophy. Ann Neurol 2012, 71(3):304-313.

20. Oetting WS, King RA. Molecular basis of albinism: mutations and polymorphisms of pigmentation genes associated with albinism. Hum Mutat. 1999;13(2):99-115.

21. Nishimasu H, Shi X. Engineered CRISPR-Cas9 nuclease with expanded targeting space. Nature. 2018;361(6408):1259-62.

22. Hutton M, Lendon CL, Rizzu P, Baker M, Froelich S, Houlden H, PickeringBrown S, Chakraverty S, Isaacs A, Grover A, et al. Association of missense and $5^{\prime}$-splice-site mutations in tau with the inherited dementia FTDP-17. Nature. 1998:393(6686):702-5.

23. Kouno T, Silvas TV, Hilbert BJ, Shandilya SMD, Bohn MF, Kelch BA, Royer WE, Somasundaran M, Kurt Yilmaz N, Matsuo H, et al. Crystal structure of APOBEC3A bound to single-stranded DNA reveals structural basis for cytidine deamination and specificity. Nat Commun. 2017;8:15024.

24. Rathore A, Carpenter MA, Demir O, Ikeda T, Li M, Shaban NM, Law EK, Anokhin D, Brown WL, Amaro RE, et al. The local dinucleotide preference of APOBEC3G can be altered from $5^{\prime}$-CC to $5^{\prime}$-TC by a single amino acid substitution. J Mol Biol. 2013;425(22):4442-54.

25. Billon P, Bryant EE, Joseph SA, Nambiar TS, Hayward SB, Rothstein R, Ciccia A. CRISPR-mediated base editing enables efficient disruption of eukaryotic genes through induction of STOP codons. Molecular Cell. 2017;67(6):106879 e1064 
26. Jiang W, Feng S, Huang S, Yu W, Li G, Yang G, Liu Y, Zhang Y, Zhang L, Hou $Y$, et al. BE-PLUS: a new base editing tool with broadened editing window and enhanced fidelity. Cell Res. 2018;28(8):855-61.

27. Lee S, Ding N, Sun Y, Yuan T, Li J, Yuan Q, Liu L, Yang J, Wang Q, Kolomeisky $A B$, et al. Single C-to-T substitution using engineered APOBEC3G-nCas9 base editors with minimum genome- and transcriptomewide off-target effects. Science Advances. 2020;6(29):eaba1773.

28. Liu Z, Chen S, Shan H, Jia Y, Chen M, Song Y, Lai L, Li Z. Efficient base editing with high precision in rabbits using YFE-BE4max. Cell Death Dis. 2020;11(1):36.

29. Tan J, Zhang F, Karcher D, Bock R. Expanding the genome-targeting scope and the site selectivity of high-precision base editors. Nat Commun. 2020; 11(1):629.

30. Jin S, Fei H, Zhu Z, Luo Y, Liu J, Gao S, Zhang F, Chen YH, Wang Y, Gao C. Rationally designed APOBEC3B cytosine base editors with improved specificity. Mol Cell. 2020. in press. https://doi.org/10.1016/j.molcel.2020.07. 005.

31. Ran FA, Cong L, Yan WX, Scott DA, Gootenberg JS, Kriz AJ, Zetsche B, Shalem O, Wu X, Makarova KS, et al. In vivo genome editing using Staphylococcus aureus Cas9. Nature. 2015;520(7546):186-91.

32. Zetsche B, Gootenberg JS, Abudayyeh OO, Slaymaker IM, Makarova KS, Essletzbichler P, Volz SE, Joung J, van der Oost J, Regev A, et al. Cpf1 is a single RNA-guided endonuclease of a class 2 CRISPR-Cas system. Cell. 2015; 163(3):759-71.

33. Lee CM, Cradick TJ, Bao G. The Neisseria meningitidis CRISPR-Cas9 system enables specific genome editing in mammalian cells. Mol Therapy. 2016; 24(3):645-54.

34. Chatterjee P, Jakimo N, Jacobson JM. Minimal PAM specificity of a highly similar SpCas9 ortholog. Science Advances. 2018;4(10):eaau0766.

35. Strecker J, Jones S, Koopal B, Schmid-Burgk J, Zetsche B, Gao L, Makarova $\mathrm{KS}$, Koonin EV, Zhang F. Engineering of CRISPR-Cas12b for human genome editing. Nat Commun. 2019;10(1):212.

36. Teng F, Cui T, Feng G, Guo L, Xu K, Gao Q, Li T, Li J, Zhou Q, Li W. Repurposing CRISPR-Cas12b for mammalian genome engineering. Cell Discov. 2018;4:63.

37. Edraki A, Mir A, Ibraheim R, Gainetdinov I, Yoon Y, Song CQ, Cao Y, Gallant J, Xue W, Rivera-Perez JA, et al. A compact, high-accuracy Cas9 with a dinucleotide PAM for in vivo genome editing. Molecular Cell. 2019;73(4): 714-26 e714.

38. Jin S, Zong Y. Cytosine, but not adenine, base editors induce genome-wide off-target mutations in rice. Science (New York, NY). 2019;364(6437):292-5.

39. Zuo E, Sun Y, Wei W, Yuan T, Ying W, Sun H, Yuan L, Steinmetz LM, Li Y, Yang $\mathrm{H}$. Cytosine base editor generates substantial off-target singlenucleotide variants in mouse embryos. Science (New York, NY). 2019; 364(6437):289-92.

40. Grunewald J, Zhou R, Garcia SP, Iyer S, Lareau CA, Aryee MJ, Joung JK. Transcriptome-wide off-target RNA editing induced by CRISPR-guided DNA base editors. Nature. 2019;569(7756):433-7.

41. Zhou C, Sun Y, Yan R, Liu Y, Zuo E, Gu C, Han L, Wei Y, Hu X, Zeng R, et al. Off-target RNA mutation induced by DNA base editing and its elimination by mutagenesis. Nature. 2019;571(7764):275-8.

42. Doman JL, Raguram A, Newby GA, Liu DR. Evaluation and minimization of Cas9-independent off-target DNA editing by cytosine base editors. Nat Biotechnol. 2020;38(5):620-8.

43. Zuo E, Sun Y, Yuan T, He B, Zhou C, Ying W, Liu J, Wei W, Zeng R, Li Y, et al. $A$ rationally engineered cytosine base editor retains high on-target activity while reducing both DNA and RNA off-target effects. Nat Methods. 2020; 17(6):600-4.

44. Yu Y, Leete TC, Born DA, Young L, Barrera LA, Lee SJ, Rees HA, Ciaramella G, Gaudelli NM. Cytosine base editors with minimized unguided DNA and RNA off-target events and high on-target activity. Nat Commun. 2020;11(1):2052.

45. Ryu SM, Koo T, Kim K, Lim K, Baek G, Kim ST, Kim HS, Kim DE, Lee H, Chung $E$, et al. Adenine base editing in mouse embryos and an adult mouse model of Duchenne muscular dystrophy. Nat Biotechnol. 2018;36(6):536-9.

46. Villiger L, Grisch-Chan HM, Lindsay H, Ringnalda F, Pogliano CB, Allegri G, Fingerhut R, Haberle J, Matos J, Robinson MD, et al. Treatment of a metabolic liver disease by in vivo genome base editing in adult mice. Nat Med. 2018;24(10):1519-25.

47. Lim CKW, Gapinske M, Brooks AK, Woods WS, Powell JE, Zeballos CM, Winter J, Perez-Pinera P, Gaj T. Treatment of a mouse model of ALS by in vivo base editing. Molecular Therapy. 2020;28(4):1177-89.
48. Levy JM, Yeh WH, Pendse N, Davis JR, Hennessey E, Butcher R, Koblan LW, Comander J, Liu Q, Liu DR. Cytosine and adenine base editing of the brain, liver, retina, heart and skeletal muscle of mice via adeno-associated viruses. Nature Biomed Eng. 2020;4(1):97-110.

49. Song Y, Yuan L, Wang Y, Chen M, Deng J, Lv Q, Sui T, Li Z, Lai L. Efficient dual sgRNA-directed large gene deletion in rabbit with CRISPR/Cas9 system. Cell Molecular Life Sci. 2016;73(15):2959-68.

50. Bae S, Park J, Kim JS. Cas-OFFinder: a fast and versatile algorithm that searches for potential off-target sites of Cas9 RNA-guided endonucleases. Bioinformatics (Oxford, England). 2014;30(10):1473-5.

51. Deng J, Chen M, Liu Z, Song Y, Sui T, Lai L, Li Z. The disrupted balance between hair follicles and sebaceous glands in Hoxc13-ablated rabbits. FASEB J. 2019;33(1):1226-34.

\section{Publisher's Note}

Springer Nature remains neutral with regard to jurisdictional claims in published maps and institutional affiliations.

Ready to submit your research? Choose BMC and benefit from:

- fast, convenient online submission

- thorough peer review by experienced researchers in your field

- rapid publication on acceptance

- support for research data, including large and complex data types

- gold Open Access which fosters wider collaboration and increased citations

- maximum visibility for your research: over $100 \mathrm{M}$ website views per year

At $\mathrm{BMC}$, research is always in progress.

Learn more biomedcentral.com/submissions 